\title{
Accidental rectal injury during a boat trip in a child: a challenge for telemedicine
}

\author{
Antonella Centonze ${ }^{1}$, Domenico Salerno ${ }^{1}$, Stellario Capillo ${ }^{1}$, Aurelio Mazzei $^{1}$, \\ Giuseppe Stranieri ${ }^{1}$, Ilaria Prosperi Porta ${ }^{2}$, Emanuele Baldassarre ${ }^{3}$ \\ ${ }^{1}$ Department of Paediatric Surgery, Pugliese-Ciaccio Hospital, Catanzaro, Italy \\ ${ }^{2}$ Department of Emergency Medicine, ASL Valle d'Aosta, Italy \\ ${ }^{3}$ Department of Andrology and Paediatric Urology, ASL Valle d'Aosta, Italy
}

We read the stimulating article recently published in your journal by Sagaro et al. [1] that reports the experience of telemedical assistance at sea of Centro Internazionale Radio Medico (CIRM) during coronavirus disease 2019 (COVID-19) pandemic. The Italian Telemedical Maritime Assistance Service (TMAS) represents the largest experience at sea in the world.

This system, actually used by cargo ships or cruise ships, could be helpful also in commoner situations, like ferries or private boats. The rationale is having first medical contact in difficult-to-manage situations, such as severe allergic crisis or trauma, equipping the boats with adequate devices.

Recently we treated the case of a 10-year-old girl affected by a perineal trauma after a fall on a wet surface with impaling on the rudder of a pleasure boat. The situation did not appear immediately critical and the crew decided to return to shore without haste.

About 8 hours after the trauma she referred to the Emergency Room with rectal bleeding and abdominal pain. She was haemodynamically stable, without visible wounds, perineal or vaginal lesions, and her urine was clear. The computed tomography showed the presence of intraperitoneal fluid and some hydro-aerated levels, with air close to the right anterolateral wall of the sigma-rectum passage. The walls of the rectum were thickened with gaseous areolas in the context. The sigmoidorectoscopy demonstrated a lesion of the rectum mucosa, suspected for perforation of the intraperitoneal rectum. At laparotomy faecal material was found in the rectovaginal space, spilling from a rectal wall lesion at the peritoneal reflection level. The perforation was repaired and a protective colostomy was done. The postoperative course was uneventful and the recanalisation was performed after 6 months. There were no long-term complications.

Penetrating anorectal injuries in children are rare and thus remain relatively under-reported in literature [2]. The commonest causes are falls on sharp objects, sexual abuse, gunshot wounds, traffic accidents, enemas, ingested foreign bodies and use of rectal thermometers.

According to Beiler et al. [3], severity of the wounds is difficult to estimate because too small lesions are not obvious or immediately visible: anyway, it's important, in terms of management and outcome, a main distinction between injuries of the intraperitoneal and extraperitoneal segments of the rectum, due to the different management [4].

Recent literature suggests individual management for anorectal lesions, in particular small and isolated anal lesions could be sutured without colostomy while more extensive injuries require faecal deviation. The prognosis of penetrating perineal lesions during childhood is good, even in cases of severe anorectal damage. Peritonism should be considered as an urgent indication for laparotomy or laparoscopy [5].

Herein could the presence of telemedicine on board be an added value? Would an early diagnostic suspicion change the outcome? A journey at sea could reserve pitfalls: inexperience, unusual accidents, absence of telephone network, and poor knowledge of on-board instrumentation. In our opinion, in this case, an early consultation in telemedicine would probably have reduced the access times to the first treatment. The future will lie in making systems such as TMAS increasingly usable. 


\section{REFERENCES}

1. Sagaro GG, Battineni G, Chintalapudi N, et al. Telemedical assistance at sea in the time of COVID-19 pandemic. Int Marit Health. 2020; 71(4): 229-236, doi: 10.5603/IMH.2020.0041, indexed in Pubmed: 33394487.

2. Vincent MV, Abel C, Duncan ND. Penetrating anorectal injuries in Jamaican children. Pediatr Surg Int. 2012; 28(11): 1101-1107, doi: 10.1007/s00383-012-3176-5, indexed in Pubmed: 23011491.

3. Beiler HA, Zachariou Z, Daum R. Impalement and anorectal injuries in childhood: A retrospective study of 12 cases. J Pediatr
Surg. 1998; 33(8): 1287-1291, doi: 10.1016/s0022-3468 (98)90170-1.

4. El Lakis MA, Rida K, Nakhle R, et al. Complex rectal and anal canal injuries secondary to unusual blunt perineal trauma. BMJ Case Rep. 2014; 2014, doi: 10.1136/bcr-2014-206060, indexed in Pubmed: 25352384.

5. Jones JG, Worthington T. Genital and anal injuries requiring surgical repair in females less than 21 years of age. J Pediatr Adolesc Gynecol. 2008; 21(4): 207-211, doi: 10.1016/j.jpag.2007.10.010, indexed in Pubmed: 18656075. 